\title{
Acceptability and tolerability of and adherence to HIV preexposure prophylaxis among Toronto gay and bisexual men: a pilot study
}

\author{
Darrell H.S. Tan MD PhD, Alexandre Schnubb, James Lawless BSc, Leah Szadkowski MSc, \\ Troy Grennan MD, James Wilton MPH, Shawn Fowler, Trevor A. Hart PhD, John Maxwell BA, \\ Janet M. Raboud $\mathrm{PhD}$
}

Abstract

Background: Preexposure prophylaxis is efficacious at preventing HIV infection, but concerns persist about adherence and sexually transmitted infections (STIs). We assessed preexposure prophylaxis acceptability, adherence and clinical outcomes in a pilot demonstration project.

Methods: HIV-uninfected adult gay and bisexual men who scored 10 or higher on a validated HIV risk score (HIV Incidence Risk Index for MSM) and reported condomless receptive anal sex were sequentially enrolled into a 1-year open-label single-arm pilot study of daily oral therapy with tenofovir disoproxil fumarate/emtricitabine in Toronto. The primary outcome was acceptability of preexposure prophylaxis. Secondary outcomes were preexposure prophylaxis adherence (4-d recall, pill count and dried blood spot analysis), HIV seroconversion, STIs and adverse events.

Results: Of the 86 men screened, 52 were enrolled. Participants were mostly young (median age 33 yr [interquartile range (IQR) 28-37 yr) white (38 [73\%]) gay (49 [94\%]) men. Preexposure prophylaxis acceptability was high: all participants reported their experience as "good" or "very good." The median adherence rate was high, at 100\% (IQR 95\%-100\%) by self-report and 96.9\% (IQR 93.4\%$98.4 \%$ ) by pill count. Dried blood spot analysis suggested that doses were taken 4-7 days/week at 88.7\% (173/195) of month 3-12 visits. No cases of HIV seroconversion occurred, but 25 participants $(48 \%)$ experienced at least 1 bacterial STI, with incidence rates per 100 person-years of $32.8,32.8,8.2$ and 8.2 for chlamydia, gonorrhea, syphilis and lymphogranuloma venereum, respectively. No adverse events led to discontinuation of prophylaxis, but the estimated glomerular filtration rate declined by $0.22 \mathrm{~mL} / \mathrm{min}$ per month.

Interpretation: Preexposure prophylaxis was associated with high adherence and acceptability and no HIV infections in this study. Frequent STIs and clinically unapparent toxic renal effects reinforce the need for ongoing vigilance. Trial registration: ClinicalTrials. gov, no. NCT02149888

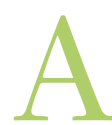

disproportionate burden of HIV infections in Canada occurs among gay, bisexual and other men who have sex with men, who account for $49.7 \%$ of prevalent infections and have a 131-fold higher risk of incident HIV than other Canadian men. ${ }^{1}$ Preexposure prophylaxis with daily oral tenofovir disoproxil fumarate/emtricitabine therapy is a biomedical HIV prevention approach that has been shown to be safe and efficacious in reducing HIV acquisition in randomized trials. ${ }^{2-5}$ As the results of these studies became available, interest increasingly turned to conducting "demonstration projects" or clinical trials addressing implementation outcomes such as adherence and real-world effectiveness. ${ }^{6-8}$ However, in surveys we conducted among stakeholders across Canada, ${ }^{9-13}$ respondents expressed concerns about the potential for suboptimal adherence, sexually transmitted infections (STIs) and toxic drug effects. Furthermore, there was uncertainty about the acceptability of preexposure prophylaxis, fuelled in part by reports of slow uptake in other settings. ${ }^{14,15}$

To address these concerns and inform broader rollout in Canada, we conducted a pilot demonstration project among gay, bisexual and other men who have sex with men in Toronto. Our primary objective was to assess the acceptability of preexposure prophylaxis at the community and individual levels, by quantifying both the volume of referrals to the study and participants' satisfaction. As secondary outcomes, we also quantified adherence, HIV seroconversion, bacterial STIs and adverse events.

Competing interests: In the past 2 years, Darrell Tan's institution has received research support for investigator-initiated research studies from Gilead Sciences and ViiV Healthcare, and Darrell Tan has been a site principal investigator for clinical trials sponsored by GlaxoSmithKline. Janet Raboud is a coinvestigator on several projects with in-kind contributions or research support from Merck \& Co. and Gilead Sciences. No other competing interests were declared.

This article has been peer reviewed.

Correspondence to: Darrell Tan, darrell.tan@gmail.com

CMAJ Open 2018. DOI:10.9778/cmajo.20180068 


\section{Methods}

\section{Study design}

PREPARATORY-5 was a 1-arm, open-label pilot demonstration project of daily preexposure prophylaxis among high-risk Toronto gay, bisexual and other men who have sex with men (NCT02149888). Study staff dispensed tenofovir disoproxil fumarate/emtricitabine $(300 / 200 \mathrm{mg}), 1$ tablet administered orally once daily over 1 year, during quarterly follow-up visits at St. Michael's Hospital, a tertiary academic hospital.

We conceived the trial as a pilot study because we planned to use the findings to inform the design of future studies. In accordance with a previously published taxonomy of reasons for conducting pilot studies, ${ }^{16}$ our study objectives corresponded to both process and scientific reasons. Our specific process-related objectives were to determine the rate of referrals for preexposure prophylaxis from community organizations as well as preexposure prophylaxis acceptability to inform potential recruitment rates for future trials. As scientific objectives, we sought to quantify key outcomes such as adherence and STIs to inform the sample size calculations for such studies.

\section{Participants}

We recruited participants through self-referral and provider referral between Oct. 16 and Dec. 30, 2014, as previously described. ${ }^{17}$ English-speaking men aged 18 years or more were eligible if they reported having sex with men, tested nonreactive on a fourth-generation HIV test (Architect antigen/antibody combo assay, Abbott Laboratories), had a creatinine clearance rate of $60 \mathrm{~mL} / \mathrm{min}$ or greater by the Modified Diet in Renal Disease formula, reported condomless receptive anal sex over the preceding 6 months and scored 10 or higher on the HIV Incidence Risk Index for MSM, a recommended cut-off value for identifying candidates for preexposure prophylaxis. ${ }^{18}$

Exclusion criteria included symptoms or signs of HIV seroconversion, use of pre- or postexposure prophylaxis within the preceding 3 months, concomitant therapy with nephrotoxic or immunomodulatory drugs, hepatitis B surface antigen positivity, high risk of osteoporosis, enrolment in another HIV prevention trial or perceived inability to adhere to the study protocol. The last criterion refers to situations in which the potential participant and the study staff together determine that the study visits required by the study protocol are not feasible for the person owing to frequency, timing and/or time commitment. Eligibility criteria were assessed via standardized interviews and blood tests done during screening.

\section{Study procedures}

Eligible men attended a baseline visit within 2 weeks and follow-up visits at months 1, 3, 6, 9 and 12 thereafter. Each visit included assessment for adverse events, Insti HIV rapid antibody testing (bioLytical Laboratories), fourth-generation HIV testing, complete blood count, determination of creatinine and phosphate levels, serologic testing for syphilis, pill count, dried blood spot collection for measurement of intraerythrocytic tenofovir disphosphate levels ${ }^{19}$ and drug dispensing. At all visits except month 1, participants also under- went urinalysis and screening for gonorrhea and chlamydia infections by means of urine nucleic acid amplification testing, and pharyngeal and rectal swabs were collected for culture. Data on STIs diagnosed at other facilities were collected by history. Every visit included personalized counselling on adherence with preexposure prophylaxis and sexual risk reduction. Electronic questionnaires assessed preexposure prophylaxis acceptability and adherence (Adult AIDS Clinical Trials Group Adherence Questionnaire ${ }^{20}$ ). Participants received \$25 compensation per visit. Each participant was also asked to attend a single adherence-support session with an experienced counsellor housed at a partner community-based organization, where one-on-one counselling was provided based on a published adherence-support intervention. ${ }^{21}$

\section{Sample size considerations}

The target sample size was 50 participants, based primarily on feasibility considerations. This sample size permitted estimation of the proportion of participants reporting high acceptability, anticipated at $80 \%-90 \%$, with reasonable precision (within 10\%).

\section{Outcome measures and statistical analysis}

We summarized demographic and clinical characteristics of participants using descriptive statistics. To assess communitylevel interest in preexposure prophylaxis, we quantified the number of individual referrals received per unit time. To assess individual-level acceptability, we calculated each participant's response to the question "Overall, how would you rate your experience on PrEP [preexposure prophylaxis]?”, averaged over all available study visits, on a Likert scale ranging from 1 ("very bad") to 5 ("very good").

We quantified adherence using self-report, pill count and intraerythocytic tenofovir diphosphate levels in dried blood spots. ${ }^{22} \mathrm{We}$ used self-report data to calculate the proportion of doses taken over the preceding 4 days and pill count data to estimate the total number of doses taken between successive study visits. We used dried blood spot data to classify the results into 4 categories: dosing 7 days/week, 4-6 days/week, 2-3 days/week or less than 2 days/week, corresponding to HIV risk reduction values of $100 \%, 100 \%$ (95\% confidence interval [CI] $86 \%$ to $100 \%), 84 \%$ (95\% CI $-24 \%$ to $99 \%)$ and $44 \%$ (95\% CI $-31 \%$ to $77 \%$ ), respectively. ${ }^{23}$ At the time that this study was designed, the relation between adherence and effectiveness of preexposure prophylaxis had not been formally quantified, which precluded specification of an evidence-based threshold for adherence a priori.

We tabulated incident STIs, converted into incidence rates per 100 person-years of follow-up. We classified clinical adverse events according to the Division of AIDS Table for Grading the Severity of Adult and Pediatric Adverse Events, ${ }^{24}$ severity and investigator-assessed probability of association with the study drug. We calculated the creatinine clearance rate at each visit using the Modified Diet in Renal Disease equation and modelled the effect of time on estimated glomerular filtration rate using a linear mixed model with a random intercept and a fixed effect of continuous time. Sexual behaviour data 
were collected but were not a protocol-defined secondary objective; they will be the subject of a separate publication.

We did not impute missing data because we achieved 93.8\% of planned follow-up. Analyses were conducted with SAS version 9.4 (SAS Institute) and R version 3.4.1 (R Foundation for Statistical Computing).

\section{Ethics approval}

Ethics approval was provided by the research ethics boards of St. Michael's Hospital, Ryerson University, the University Health Network and the University of Toronto. All participants provided written informed consent before any study activities.

\section{Results}

\section{Participant flow and recruitment}

Our process-related evaluations showed that, during the 75-day recruitment period, community-based organizations referred 115 people, and our electronic advertisements generated 1518 click-throughs. These referrals generated 165 unique inquiries about trial participation (2.2/d) from the community.

Of the 86 men screened for participation, 52 were eligible and were enrolled (Figure 1). Those deemed ineligible were not significantly different from enrolled participants with respect to referral source (self- v. community-referred), age, ethnicity, sexual orientation, income and education (data not shown). Of the 52 participants enrolled, $43(83 \%)$ were retained for the full year, and 1, 2 and 6 participants left the study early, after their month 3, 6 and 9 visits respectively, producing 48.75 personyears of follow-up overall. Baseline characteristics were similar for the 43 retained participants (Table 1) and the 9 who left

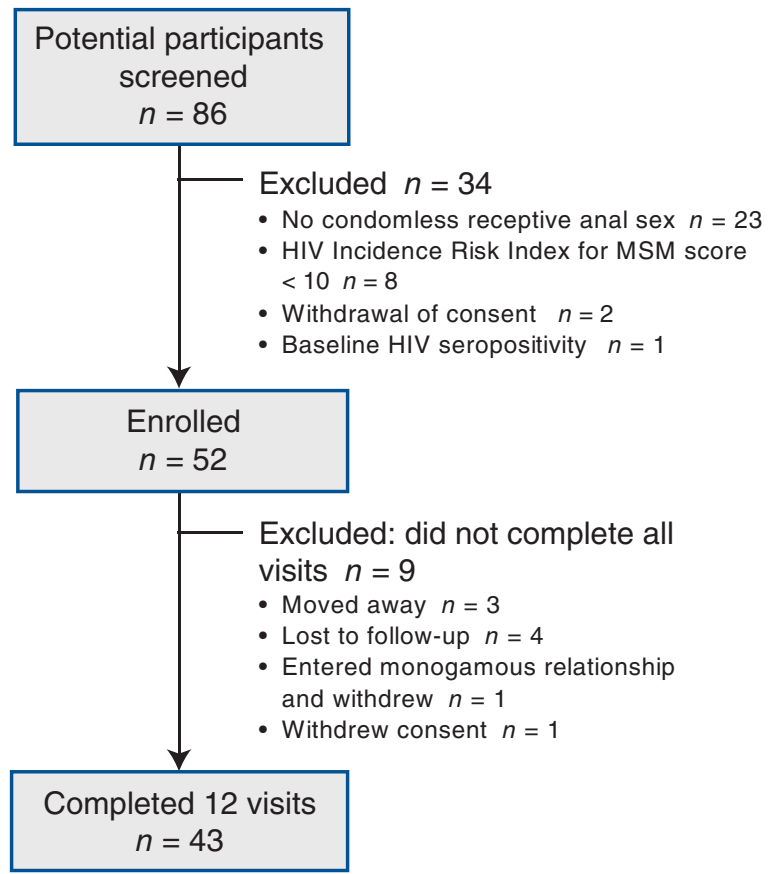

Figure 1: Flow diagram showing participant selection.
Table 1: Participant sociodemographic and behaviour characteristics

\begin{tabular}{cc}
\hline & No. $(\%)$ of \\
characteristic & $n=52$ \\
& $n=52$
\end{tabular}

Age, yr, median (IQR)

$33(28-37)$

Race

\begin{tabular}{|lc|}
\hline White & $38(73)$ \\
\hline Asian & $4(8)$ \\
\hline Latino & $3(6)$ \\
\hline Middle Eastern & $3(6)$ \\
\hline Other† & $4(8)$ \\
\hline Sexual orientation & \\
\hline
\end{tabular}

Gay 49 (94)

Bisexual‡ $3(6)$

Education

High school or some postsecondary $14(27)$

Undergraduate 23 (44)

Graduate 15 (29)

Annual income, \$

$\leq 20000$

$20001-40000$

$40001-60000$

$60001-80000$

$80001-100000$

$>100000$

No. of prescription medications, median (IQR) $0(0-1)$

No. of supplements, median (IQR) $1(0-3)$

Current smoker 13 (25)

Previous sexually transmitted infection diagnosis

\begin{tabular}{|lc|}
\hline Gonorrhea & $21(40)$ \\
\hline Chlamydia & $24(46)$ \\
\hline Syphilis & $10(19)$ \\
\hline Any bacterial sexually transmitted infection & $36(69)$ \\
\hline Baseline HIV Incidence Risk Index for MSM & $29(22.5-33.0)$
\end{tabular}
score, median (IQR)

Recreational drug use in prior $3 \mathrm{mo}$

Amphetamines (crystal) 9 (17)

"Poppers" 36 (69)

Baseline sexual behaviours in prior $6 \mathrm{mo}$, median (IQR)

\begin{tabular}{lc|}
\hline No. of partners & $18(12-30.5)$ \\
\hline No. of HIV-positive partners & $1(0-3)$ \\
\hline Condomless receptive anal sex, no. of times & $5(2-15)$ \\
$\begin{array}{l}\text { Condomless receptive anal sex with } \\
\text { HIV-positive partner, no. of times }\end{array}$ & $0(0-0)$ \\
Condomless insertive anal sex, no. of times & $5(2-12.5)$ \\
\hline $\begin{array}{l}\text { Condomless insertive anal sex with } \\
\text { HIV-positive partner, no. of times }\end{array}$ & $0(0-3)$ \\
$\begin{array}{l}\text { Note: IQR }=\text { interquartile range. } \\
\text { Except where noted otherwise. } \\
\text { Includes participants who identified as mixed }(n=2), \text { black }(n=1) \text { or First Nations }(n=1) . \\
\text { Includes } 1 \text { participant who identified as pansexual. }\end{array}$
\end{tabular}


early (data not shown). Study visits were conducted between Nov. 10, 2014, and June 28, 2016.

\section{Baseline characteristics}

Most of the participants identified as gay (49 [94\%]) white $(38[73 \%])$ men with an undergraduate degree or higher (38 [73\%]). The median age was 33 (interquartile range [IQR] 28-37) years (Table 1). The median number of prescription medications taken was 0 (IQR 0-1), but the median number of supplements taken was 1 (IQR 0-3). Most participants (36 [69\%]) had a prior history of 1 or more bacterial STIs. The median number of sexual partners over the preceding 6 months was 18 (IQR 12-30.5), and the median number of condomless receptive and insertive anal sex acts was $5(2-15)$ and $5(2-12.5)$, respectively.

\section{Outcomes}

Additional process-related evaluations showed that individuallevel preexposure prophylaxis acceptability was high. After we averaged data for each participant over all follow-up visits for which responses were available $(5,4$ or 3 visits for $81 \%, 12 \%$ and $8 \%$ of participants, respectively), $100 \%$ of participants rated their experience with preexposure prophylaxis as 4 ("good") or 5 ("very good"), and the median overall response was 4.8 (IQR 4.4-5.0).

Overall adherence to preexposure prophylaxis was high, whether assessed by self-report, pill count or dried blood spot analysis (Table 2). The median adherence rate was $100 \%$ (IQR 95\%-100\%) by self-report and 96.9\% (IQR 93.4\%98.4\%) by pill count. Intraerythrocytic tenofovir diphosphate levels were consistent with dosing on 7 days, 4-6 days, $2-3$ days and less than 2 days per week, at $50.6 \%, 36.8 \%$, $9.7 \%$ and $2.8 \%$ of all study visits, respectively. After we removed month 1 data, since tenofovir diphosphate may not have reached steady state inside erythrocytes at this point, ${ }^{22}$ these figures increased to $58.5 \%, 30.3 \%, 8.7 \%$ and $2.6 \%$, respectively.

There were no cases of HIV seroconversion. However, the burden of bacterial STIs was high, with 40 confirmed infections occurring in 25 participants (48\%) (Table 3). Fifteen
[60\%]) of these 25 men had a prior history of an STI. In addition, there were 6 episodes of nonspecific urethritis treated empirically at other facilities, for which gonorrhea and chlamydia testing reportedly gave negative results.

\section{Adverse events}

Of the 185 adverse events overall (Table 4), 37 (20\%) were at least possibly related to the study drug, but all were mild (30 [81\%]) or moderate (7 [19\%]) in severity, and none led to discontinuation of prophylaxis. The most common adverse events at least possibly related to study drug were nausea, diarrhea and headache, occurring in $11(21 \%), 6(12 \%)$ and 6 (12\%) participants, respectively. The only serious adverse event was hospital admission for severe but self-limited infectious colitis, for which no specific cause was identified. The other 2 adverse events graded as severe included 1 episode each of lymphogranuloma venereum and stress. All 3 severe adverse events were deemed unrelated to the study drug.

We observed 1 grade 2 and 3 grade 1 instances of creatinine level elevation, all of which resolved spontaneously without the need to interrupt the study drug. The creatinine clearance rate appeared to change by -0.22 ( $95 \%$ CI -0.45 to 0.01 ) $\mathrm{mL} / \mathrm{min}$ per month of follow-up in a generalized linear mixed model, but this change was of borderline statistical significance $(p=0.06)$.

\section{Interpretation}

In this pilot demonstration project, we observed high acceptability of preexposure prophylaxis at the community and individual levels, excellent adherence, a favourable adverse event profile and no cases of seroconversion over 48.75 personyears of follow-up. The high adherence is important because adherence is the key predictor of preexposure prophylaxis effectiveness. ${ }^{2,23,25}$ However, STIs were common, as seen elsewhere, ${ }^{26,27}$ and the creatinine clearance rate appeared to decline by $0.22 \mathrm{~mL} / \mathrm{min}$ per month. Our process-related findings confirm the feasibility of preexposure prophylaxis in this population, and our scientific findings highlight the need for ongoing attention to STIs and clinically unapparent toxic

Table 2: Adherence by measurement technique

\begin{tabular}{|c|c|c|c|c|c|c|c|c|}
\hline \multirow[b]{2}{*}{ Month } & \multirow[b]{2}{*}{$\begin{array}{l}\text { No. of } \\
\text { participants }\end{array}$} & \multicolumn{2}{|c|}{$\%$ of doses taken, median (IQR) } & \multicolumn{5}{|c|}{$\begin{array}{l}\text { Tenofovir disoproxil fumarate/emtricitabine level, fmol/punch*; } \\
\text { no. (\%) of participants }\end{array}$} \\
\hline & & $\begin{array}{l}\text { Self-reported } 4-d \\
\text { recall }\end{array}$ & Pill count & Median (IQR) & $\leq 349$ & $350-699$ & $700-1249$ & $\geq 1250$ \\
\hline 1 & 52 & $100(100-100)$ & $100(96.4-100)$ & $930(746.5-1199)$ & $2(4)$ & $7(13)$ & $32(62)$ & $11(21)$ \\
\hline 3 & 52 & $100(100-100)$ & $98.7(93.7-100)$ & $1341(1062-1555.5)$ & $0(0)$ & $4(8)$ & $18(35)$ & $30(58)$ \\
\hline 6 & 51 & $100(100-100)$ & $98.9(92.9-100)$ & $1432(1068-1847)$ & $0(0)$ & $4(8)$ & $15(29)$ & $32(63)$ \\
\hline 9 & 48 & $100(100-100)$ & $98.9(95.0-100)$ & $1392.5(1141-1662.5)$ & $1(2)$ & $3(6)$ & $11(23)$ & $33(69)$ \\
\hline 12 & 42 & $100(95.0-100)$ & $98.0(90.1-100)$ & 1191.5 (777-1527) & $2(5)$ & $6(14)$ & $15(36)$ & $19(45)$ \\
\hline
\end{tabular}




\begin{tabular}{|lcc|}
\hline Table 3: Incident sexually transmitted infections \\
\hline Infection & $\begin{array}{c}\text { No. of } \\
\text { cases }\end{array}$ & $\begin{array}{c}\text { Incidence per } \\
100 \text { person-years of } \\
\text { follow-up }(95 \% \mathrm{Cl})\end{array}$ \\
\hline Gonorrhea & 16 & $32.8(19.4$ to 52.2$)$ \\
\hline Urethral & 5 & \\
\hline Pharyngeal & 2 & \\
\hline Rectal & 6 & $32.8(19.4$ to 52.2$)$ \\
\hline Multiple sites & 3 & \\
\hline Chlamydia & 16 & \\
\hline Urethral & 7 & \\
\hline Pharyngeal & 1 & \\
\hline Rectal & 8 & \\
\hline $\begin{array}{l}\text { Lymphogranuloma } \\
\text { venereum (rectal) }\end{array}$ & 4 & \\
\hline Syphilis & 4 & \\
\hline Note: Cl = confidence interval. & & \\
\hline
\end{tabular}

drug effects. Our results are broadly consistent with those of other studies, ${ }^{28}$ and our estimates regarding referral rates, adherence and STIs will be helpful in the design of future studies.

Our inclusion criteria were selected to include gay, bisexual and other men who have sex with men at very high risk for HIV. Specifically, all participants reported condomless receptive anal sex within the preceding 6 months and scored high on a validated risk score (HIV Incidence Risk Index for MSM). ${ }^{18}$ The sample thus represents a population that should be prioritized for the rollout of preexposure prophylaxis in order to maximize both its public health impact and its cost-effectiveness.

Because our study lacked a comparison group, it was not possible to determine to what extent preexposure prophylaxis was causally related to the high burden of STIs observed. Previous studies have given mixed findings on this topic. A metaanalysis summarizing cohorts of men using versus not using preexposure prophylaxis showed incidence rate ratios of 25.3, 11.2 and 44.6 for gonorrhea, chlamydia and syphilis, respectively..$^{29}$ However, since these infections are also an important marker of HIV risk, these results are heavily confounded by indication; high incidence could alternatively signify that programs have been successful at identifying those in greatest need. That $69 \%$ of our study participants had a prior bacterial STI at study entry supports this notion. High rates could also be partly attributable to increased screening. The PROUD (Pre-exposure Prophylaxis to Prevent the Acquisition of HIV-1 Infection) study showed that, after adjustment for the frequency of testing, the odds of any bacterial STI was similar to that for control participants (odds ratio 1.07, 95\% CI 0.78 to 1.46$).{ }^{27}$ Additional control strategies for STIs are urgently needed. ${ }^{30}$

Although concern about side effects has been reported as the most common reason for not wanting to use preexposure

\section{Table 4: Adverse events}

\begin{tabular}{|lcc|}
\hline Adverse event & $\begin{array}{c}\text { No. (\%) of } \\
\text { participants } \\
n=52\end{array}$ & $\begin{array}{c}\text { No. (\%) of } \\
\text { events } \\
n=185\end{array}$ \\
\hline Any & $47(90)$ & - \\
\hline Grade 1 (mild) & $34(65)$ & $144(77.8)$ \\
\hline Grade 2 (moderate) & $10(19)$ & $38(20.5)$ \\
\hline Grade 3 (severe) & $3(6)$ & $3(1.6)$ \\
\hline Any serious & $1(2)$ & $1(0.5)$ \\
\hline By relation to study drug & $14(27)$ & $26(14.0)$ \\
\hline Possibly & $9(17)$ & $11(5.9)$ \\
\hline Probably & $11(21)$ & $12(6.5)$ \\
\hline At least possibly related to study drug & $6(3.2)$ \\
\hline Nausea & $6(12)$ & $6(3.2)$ \\
\hline Diarrhea & $6(12)$ & $5(2.7)$ \\
\hline Headache & $5(10)$ & $2(1.1)$ \\
\hline Fatigue & $2(4)$ & $2(1.1)$ \\
\hline Bloating & $2(4)$ & $1(0.5)$ \\
\hline Vivid dreams & $1(2)$ & $1(0.5)$ \\
\hline Anorexia & $1(2)$ & $1(0.5)$ \\
\hline Difficulty sleeping & $1(2)$ & \\
\hline Flatulence & $1(2)$ & \\
\hline Itching & & \\
\hline
\end{tabular}

prophylaxis in acceptability studies, ${ }^{9,31}$ adverse events in our cohort were minimal and were generally restricted to mild gastrointestinal symptoms that resolved spontaneously. These findings are consistent with a systematic review of preexposure prophylaxis clinical trials, in which the risk of adverse events was similar to that with placebo (relative risk 1.01, 95\% CI 0.99 to 1.03$).{ }^{32}$ However, we did observe clinically unapparent declines in the creatinine clearance rate, at $-0.22 \mathrm{~mL} /$ min per month, or $-2.64 \mathrm{~mL} / \mathrm{min}$ per year. This is greater than the age-related decline in glomerular filtration rate observed in healthy adults, estimated at roughly 0.75 $0.97 \mathrm{~mL} / \mathrm{min}$ per year. ${ }^{33,34}$ Our findings are consistent with those from randomized trials, in which preexposure prophylaxis has been associated with decreases in estimated glomerular filtration rate that were roughly $1 \mathrm{~mL} / \mathrm{min}$ per year greater than seen with placebo. ${ }^{35-39}$ Importantly, such changes in renal function are felt to be reversible. ${ }^{40}$

\section{Limitations}

Our pilot study has limitations that warrant consideration. First, it was not comparative, and our sample size was modest. However, our purpose was to conduct descriptive analyses only, to inform the design of future studies by addressing specific process-related and scientific objectives. Second, at the time that this study was designed, the relation between preexposure prophylaxis adherence and effectiveness among gay, bisexual and other men who have sex with men had not been formally quantified, which precluded us from specifying an 
evidence-based threshold for adherence a priori. Nevertheless, we found generally favourable adherence results, for several potential reasons. Most participants were already taking at least 1 supplement at study entry, which suggests experience with regular pill-taking and high health-seeking behaviour, and our adherence-support intervention may have further bolstered adherence. Third, we included only English-speaking participants. Fourth, 34 of the 86 men screened were ineligible for the study, mostly because of not recently having had condomless receptive anal sex, which raises the possibility of selection bias. However, the demographic characteristics of included and excluded people were similar, such that our findings are reflective of men at high risk who met our eligibility criteria. Finally, because we recruited participants in a context where preexposure prophylaxis was not widely available, our participants could be considered "early adopters" 41 and thus may not represent the broader population of at-risk gay, bisexual and other men who have sex with men in Canada. Health Canada granted regulatory approval for preexposure prophylaxis only in February 2016, and public reimbursement became available in Ontario only in September 2017.

\section{Conclusion}

Preexposure prophylaxis was associated with high adherence and acceptability and no cases of seroconversion in this study. Our findings support the broader rollout of preexposure prophylaxis for at-risk gay, bisexual and other men who have sex with men in this Canada and in similar industrialized settings. However, changes in sexual behaviour, frequent STIs and clinically unapparent effects on renal function reinforce the need for ongoing vigilance. Furthermore, these data were collected during 2014-2016, and outcomes related to preexposure prophylaxis may continue to evolve. It will be important to continually monitor outcomes as public drug coverage and community uptake increase and as clinical practice guidelines ${ }^{42}$ lead to greater prescribing. As such, the results of our pilot study have been used to perform sample size calculations for the Ontario PrEP Cohort Study, which will monitor these and related outcomes across the province in the coming years and may similarly inform the design of interventional studies in the future.

\section{References}

1. Yang, Q, Ogunnaike-Cooke S, Yan P, et al. Comparison of HIV incidence rates among key populations in Canada, 2011. Proceedings of the 20th International AIDS Conference; 2014 July 20-25; Melbourne, Australia.

2. Grant RM, Lama JR, Anderson PL, et al.; iPrEx Study Team. Preexposure chemoprophylaxis for HIV prevention in men who have sex with men. $N$ Engl 7 Med 2010;363:2587-99.

3. Baeten JM, Donnell D, Ndase P, et al.; Partners PrEP Study Team. Antiretroviral prophylaxis for HIV prevention in heterosexual men and women. $N$ Engl 7 Med 2012;367:399-410.

4. Thigpen MC, Kebaabetswe PM, Paxton LA, et al.; TDF2 Study Group. Antiretroviral preexposure prophylaxis for heterosexual HIV transmission in Botswana. N Engl 7 Med 2012;367:423-34.

5. Choopanya K, Martin M, Suntharasamai P, et al.; Bangkok Tenofovir Study Group. Antiretroviral prophylaxis for HIV infection in injecting drug users in Bangkok, Thailand (the Bangkok Tenofovir Study): a randomised, doubleblind, placebo-controlled phase 3 trial. Lancet 2013;381:2083-90.

6. Guidance on oral pre-exposure oral prophylaxis (PrEP) for serodiscordant couples, men and transgender women who bave sex with men at high risk of HIV: recommendations for use in the context of demonstration projects. Geneva: World Health Organization; 2012.
7. AIDS Foundation of Chicago; AVAC: Global Advocacy for HIV Prevention; BlackAIDS Institute; International Rectal Microbicide Advocates; National Minority AIDS Council; Project Inform; San Francisco AIDS Foundation. PrEP: roadmap to the real world - establishing the real-world effectiveness of PrEP through demonstration projects. San Francisco: Project Inform; 2011.

8. Cahill S. Re-exposure prophylaxis for HIV prevention: moving toward implementation [policy focus]. 2nd ed. Boston: Fenway Institute; 2012.

9. Wilton J, Kain T, Fowler S, et al. Use of an HIV risk screening tool to identify optimal candidates for PrEP scale-up among men who have sex with men in Toronto, Canada: disconnect between objective and subjective HIV risk. 7 Int AIDS Soc 2016;19:20777.

10. Leonardi M, Lee E, Tan DH. Awareness of, usage of and willingness to use HIV pre-exposure prophylaxis among men in downtown Toronto, Canada. Int 7 STD AIDS 2011;22:738-41.

11. Sharma M, Wilton J, Senn H, et al. Preparing for PrEP: perceptions and readiness of Canadian physicians for the implementation of HIV pre-exposure prophylaxis. PLoS One 2014;9:e105283.

12. Senn H, Wilton J, Sharma M, et al. Knowledge of and opinions on HIV preexposure prophylaxis among front-line service providers at Canadian AIDS service organizations. AIDS Res Hum Retroviruses 2013;29:1183-9.

13. Yoong D, Naccarato M, Sharma $M$, et al. Preparing for pre-exposure prophylaxis: perceptions and readiness of Canadian pharmacists for the implementation of HIV pre-exposure prophylaxis. Int 7 STD AIDS 2016;27:608-16.

14. Cáceres CF, O'Reilly KR, Mayer KH, et al. PrEP implementation: moving from trials to policy and practice. F Int AIDS Soc 2015;18(Suppl 3):20222.

15. Mayer KH, Hosek S, Cohen S, et al. Antiretroviral pre-exposure prophylaxis implementation in the United States: a work in progress. 7 Int AIDS Soc 2015; 18(Suppl 3):19980.

16. Thabane L, Ma J, Chu R, et al. A tutorial on pilot studies: the what, why and how. BMC Med Res Methodol 2010;10:1.

17. Wilton J, Noor SW, Schnubb A, et al. High HIV risk and syndemic burden regardless of referral source among MSM screening for a PrEP demonstration project in Toronto, Canada. BMC Public Health 2018;18:292.

18. Smith DK, Pals SL, Herbst JH, et al. Development of a clinical screening index predictive of incident HIV infection among men who have sex with men in the United States. 7 Acquir Immune Defic Syndr 2012;60:421-7.

19. Bushman LR, Kiser JJ, Rower JE, et al. Determination of nucleoside analog mono-, di-, and tri-phosphates in cellular matrix by solid phase extraction and ultra-sensitive LC-MS/MS detection. F Pharm Biomed Anal 2011;56:390-401.

20. Chesney MA, Ickovics JR, Chambers DB, et al. Self-reported adherence to antiretroviral medications among participants in HIV clinical trials: the AACTG adherence instruments. Patient Care Committee \& Adherence Working Group of the Outcomes Committee of the Adult AIDS Clinical Trials Group (AACTG). AIDS Care 2000;12:255-66.

21. Amico KR, McMahan V, Goicochea P, et al. Supporting study product use and accuracy in self-report in the iPrEx study: next step counseling and neutral assessment. AIDS Behav 2012;16:1243-59.

22. Castillo-Mancilla JR, Zheng JH, Rower JE, et al. Tenofovir, emtricitabine, and tenofovir diphosphate in dried blood spots for determining recent and cumulative drug exposure. AIDS Res Hum Retroviruses 2013;29:384-90.

23. Grant RM, Anderson PL, McMahan V, et al.; iPrEx study team. Uptake of pre-exposure prophylaxis, sexual practices, and HIV incidence in men and transgender women who have sex with men: a cohort study. Lancet Infect Dis 2014;14:820-9.

24. Division of AIDS Table for Grading the Severity of Adult and Pediatric Adverse Events. Version 1.0. Bethesda (MD): DAIDS Regulatory Support Center; 2004 (updated August 2009). Available: https://rsc.tech-res.com/docs/ default-source/safety/table_for_grading_severity_of_adult_pediatric_adverse_ events.pdf (accessed 2014 July 4).

25. Landovitz RJ. Preexposure prophylaxis for HIV prevention: what we know and what we still need to know for implementation. Top Antivir Med 2015;23:85-90.

26. Liu AY, Cohen SE, Vittinghoff E, et al. Preexposure prophylaxis for HIV infection integrated with municipal- and community-based sexual health services. FAMA Intern Med 2016;176:75-84.

27. McCormack S, Dunn DT, Desai M, et al. Pre-exposure prophylaxis to prevent the acquisition of $\mathrm{HIV}-1$ infection (PROUD): effectiveness results from the pilot phase of a pragmatic open-label randomised trial. Lancet 2016;387:53-60.

28. Riddell J IV, Amico KR, Mayer KH. HIV preexposure prophylaxis: a review. 7AMA 2018, 319:1261-8.

29. Kojima N, Davey DJ, Klausner JD. Pre-exposure prophylaxis for HIV infection and new sexually transmitted infections among men who have sex with men. AIDS 2016;30:2251-2.

30. Report on sexually transmitted infections in Canada: 2013-2014. Ottawa: Centre for Communicable Diseases and Infection Control, Infectious Disease Prevention and Control Branch, Public Health Agency of Canada; 2017.

31. Peng P, Su S, Fairley CK, et al. A global estimate of the acceptability of preexposure prophylaxis for HIV among men who have sex with men: a systematic review and meta-analysis. AIDS Behav 2018;22;1063-74.

32. Fonner VA, Dalglish SL, Kennedy CE, et al. Effectiveness and safety of oral HIV preexposure prophylaxis for all populations. AIDS 2016;30:1973-83.

33. Lindeman RD, Tobin J, Shock NW. Longitudinal studies on the rate of decline in renal function with age. 7 Am Geriatr Soc 1985;33:278-85. 
34. Cohen E, Nardi Y, Krause I, et al. A longitudinal assessment of the natural rate of decline in renal function with age. 7 Nephrol 2014;27:635-41.

35. Yacoub R, Nadkarni GN, Weikum D, et al. Elevations in serum creatinine with tenofovir-based HIV pre-exposure prophylaxis: a meta-analysis of randomized placebo-controlled trials. 7 Acquir Immune Defic Syndr 2016;71:e115-8.

36. Mirembe BG, Kelly CW, Mgodi N, et al.; MTN-003B Protocol Team. Bone mineral density changes among young, healthy African women receiving oral tenofovir for HIV preexposure prophylaxis. 7 Acquir Immune Defic Syndr 2016; 71:287-94.

37. Solomon MM, Lama JR, Glidden DV, et al.; iPrEx Study Team. Changes in renal function associated with oral emtricitabine/tenofovir disoproxil fumarate use for HIV pre-exposure prophylaxis. AIDS 2014;28:851-9.

38. Martin M, Vanichseni S, Suntharasamai P, et al.; Bangkok Tenofovir Study Group. Renal function of participants in the Bangkok tenofovir study Thailand, 2005-2012. Clin Infect Dis 2014;59:716-24.

39. Mugwanya KK, Wyatt C, Celum C, et al.; Partners PrEP Study Team. Changes in glomerular kidney function among HIV-1-uninfected men and women receiving emtricitabine-tenofovir disoproxil fumarate preexposure prophylaxis: a randomized clinical trial. FAMA Intern Med 2015;175:246-54.

40. Mugwanya KK, Wyatt C, Celum C, et al.; Partners PrEP Study Team. Reversibility of glomerular renal function decline in HIV-uninfected men and women discontinuing emtricitabine-tenofovir disoproxil fumarate pre-exposure prophylaxis. 7 Acquir Immune Defic Syndr 2016;71:374-80.

41. Dearing JW, Kee KF. Historical roots of dissemination and implementation science. In: Brownson RC, Colditz GA, Proctor EK, editors. Dissemination and implementation research in bealth: translating science to practice. New York: Oxford University Press; 2012:55-71.

42. Tan DHS, Hull MW, Yoong D, et al.; Biomedical HIV Prevention Working Group of the CIHR Canadian HIV Trials Network. Canadian guideline on HIV pre-exposure prophylaxis and nonoccupational postexposure prophylaxis. CMA7 2017;189:E1448-58.

Affiliations: Division of Infectious Diseases (Tan, Schnubb, Lawless), St. Michael's Hospital; Department of Medicine (Tan), University of Toronto; Toronto General Hospital Research Institute (Tan, Raboud) and Biostatistics Research Unit (Szadkowski), University Health Network, Toronto, Ont.; British Columbia Centre for Disease Control (Grennan); Division of Infectious Diseases (Grennan), University of British Columbia, Vancouver, BC; Ontario HIV Treatment Network (Wilton); Hassle Free Clinic (Fowler); Department of Psychology (Hart), Ryerson University; Dalla Lana School of Public Health (Hart, Raboud), University of Toronto; AIDS Committee of Toronto (Maxwell), Toronto, Ont.

Contributors: Darrell Tan conceived, led and oversaw all aspects of the study. Darrell Tan, Troy Grennan, James Wilton, Shawn Fowler, Trevor Hart and John Maxwell designed the study. Darrell Tan, Alexandre Schnubb and James Lawless recruited the participants and acquired the data. Darrell Tan and Janet Raboud planned the study analysis, and Darrell Tan, Leah Szadkowski and Janet Raboud analyzed the data. Darrell Tan drafted the manuscript. All of the authors provided input into the study design, critically reviewed the manuscript for important intellectual content, gave final approval of the version to be published and agreed to be accountable for all aspects of the work.

Funding: This work was supported by grants from the Ontario HIV Treatment Network (OHTN) and the Canadian Institutes of Health Research (CIHR). Study drug was provided by Gilead Sciences Inc. Rapid HIV testing kits were provided by bioLytical Laboratories Inc. Darrell Tan is supported by a New Investigator Award from the CIHR/OHTN, Trevor Hart is supported by an OHTN Applied HIV Research Chair, and Janet Raboud was supported by an OHTN Chair in Biostatistics. The funders had no role in the study design, data collection, analysis or interpretation, or manuscript writing.

Supplemental information: For reviewer comments and the original submission of this manuscript, please see www.cmajopen.ca/content/6/4/ E611/suppl/DC1. 